\title{
HOLONOMY AND THE LIE ALGEBRA OF INFINITESIMAL MOTIONS OF A RIEMANNIAN MANIFOLD
}

\author{
BY \\ BERTRAM KOSTANT
}

Introduction. .1. Let $M$ be a differentiable manifold of class $C^{\infty}$. All tensor fields discussed below are assumed to be of class $C^{\infty}$. Let $X$ be a vector field on $M$. If $X$ vanishes at a point $o \in M$ then $X$ induces, in a natural way, an endomorphism $a_{X}$ of the tangent space $V_{o}$ at $o$. In fact if $y \in V_{o}$ and $Y$ is any vector field whose value at $o$ is $y$, then define $a_{X} y=[X, Y]_{o}$. It is not hard to see that $[X, Y]_{o}$ does not depend on $Y$ so long as the value of $Y$ at $o$ is $y$.

Now assume $M$ has an affine connection or as we shall do in this paper, assume $M$ is Riemannian and that it possesses the corresponding affine connection. One may now associate to $X$ an endomorphism $a_{X}$ of $V_{o}$ for any point $o \in M$ which agrees with the above definition and which heuristically indicates how $X$ "winds around $o$ " by defining for $v \in V_{o}, a_{X} v=-\nabla_{v} X$, where $\nabla_{v}$ is the symbol of covariant differentiation with respect to $v$.

$X$ is called an infinitesmal motion if the Lie derivative of the metric tensor with respect to $X$ is equal to zero. This is equivalent to the statement that $a_{X}$ is skew-symmetric (as an endomorphism of $V_{o}$ where the latter is provided with the inner product generated by the metric tensor) for all $o \in M$.

$\S 1$ contains definitions and formulae which will be used in the remaining sections.

In $\$ 2$ we consider $\mathfrak{g}$, the space of all infinitesimal motions on $M . \mathfrak{g}$ is a finite dimensional Lie algebra under the usual bracket operation for vector fields. Each element $X \in g$ is uniquely determined over $M$ by the value $x$ of $X$ and $a_{X}$ at any single point $o \in M$. These known facts and a statement giving $[X, Y]_{o}$ and $a_{[X, Y]}$ for $X, Y \in \mathfrak{g}$ (and consequently giving the structure of $\mathfrak{g}$ ) in terms of $x, y, a_{X}, a_{Y}$ and the curvature tensor at $o$, generalizing that given by E. Cartan when $M$ is symmetric, are contained in Theorem 2.3 of this section.

In $\S 3$ we consider $\xi_{0}$ the Lie algebra of the restricted homogeneous holonomy group at $o \in M$. ( $\varepsilon_{o}$ is a Lie algebra of skew-symmetric endomorphisms of $V_{o}$.) We are interested in the question as to when $a_{X} \in z_{o}$ for $X \in \mathfrak{g}$. As Euclidean space clearly illustrates, this is not true in general. When $x=0$, Lichnerowicz [12] has shown that it is true if $B_{o}$ acts irreducibly on $V_{o}$ ( $M$ is thereby called irreducible as this is independent of $o$ ) and the Ricci tensor does not vanish. It is true under these conditions whether or not $x=0$. It is the main result of this section that this is true $\left(a_{X} \in 8_{o}\right)$ whenever $M$ is compact.

Received by the editors March 20, 1955. 
In $\S 4$ we consider $\mathfrak{h}_{0}$ the Lie algebra of skew-symmetric endomorphisms of $V_{o}$ generated by all $a_{X}$ for $X \in \mathfrak{g}$. One knows that in general $\mathfrak{h}_{o} \subseteq n\left(\xi_{o}\right)$ where $n\left(\xi_{o}\right)$ is the normalizer of $\boldsymbol{z}_{o}$ in the Lie algebra, $\mathfrak{a}_{o}$, of all skew-symmetric endomorphisms of $V_{o}$. In this section we take up the reverse question of $\S 3$, namely, when does $\varepsilon_{0} \subseteq \mathfrak{h}_{o}$. We show that this holds whenever $g$ is sufficiently $\mathrm{big}$, i.e. when $\mathfrak{g}$ is transitive in the sense that for any vector $v \in V_{o}$ at any point $o \in M$ there exists $X \in \mathfrak{g}$ such that $x=v$. This is of course true whenever $M$ is any open submanifold of a homogeneous space. It is then the principal result of this section that $\boldsymbol{B}_{0}=\mathfrak{h}_{0}$ when $M$ is a compact homogeneous space.

1. Preliminaries. .1. Let $M$ be a Riemannian manifold of class $C^{\infty}$. By this we mean that not only is $M$ a differentiable manifold of class $C^{\infty}$ but that the metric tensor is likewise of class $C^{\infty}$. Similarly, without any mention to the contrary all tensor fields considered in this paper are assumed to be defined on all of $M$ and are of class $C^{\infty}$.

We adopt the following notations:

$(\alpha) o$ is an arbitrary point of $M$,

( $\beta) V_{o}$ is the tangent space at $o$,

( $\gamma$ ) $X, Y, Z$ are vector fields on $M$, and unless specified otherwise $x, y, z$ are, respectively, the values of these vector fields at $o$,

$(\delta) S$ is an arbitrary tensor field and $s$ its value at $o$,

(द) $u, v$, are arbitrary vectors.

.2. For any $o \in M$ we define for the present $V_{o}^{1}=V_{o}$ and $V_{o}^{-1}=V_{o}^{*}$ (the dual of $\left.V_{o}\right)$ and where $\gamma$ is the $k$-tuple $\left(\gamma_{1}, \gamma_{2}, \cdots, \gamma_{k}\right), \gamma_{i}= \pm 1, i=1,2, \cdots$, $n$, define $V_{o}^{\gamma}=V_{o}^{\gamma_{1}} \otimes \cdots \otimes V_{o}^{\gamma}$. Then the direct sum

$$
\mathcal{T}\left(V_{o}\right)=\sum_{\gamma} V_{o}^{\gamma}
$$

is of course the mixed tensor algebra at $o \in M$.

If $\mathcal{T}$ is the algebra of all mixed tensor fields on $M$ let us consider any linear transformation $D_{o}$ from $\mathcal{T}$ into $\mathcal{T}\left(V_{o}\right)$ which satisfies

(1) $D_{o}\left(S_{1} S_{2}\right)=s_{1} D_{o}\left(S_{2}\right)+D_{o}\left(S_{1}\right) s_{2}$,

(2) $D_{o}$ preserves tensor type,

(3) $D_{o}\left(S^{C}\right)=D_{o}(S)^{c}$

(where $C$ designates any specific contraction). We shall call such a linear transformation a $\tau$-differentiation at $o \in M$.

An example of a $\mathcal{\tau}$-differentiation at $o$, with which we shall be concerned, is given by covariant differentiation $\nabla_{v}$ of $\mathcal{T}$ by a vector $v \in V_{o}$.

Where $f$ is a function $\nabla_{v} f$ is just $v f$, i.e. differentiation of $f$ by the vector $v$. However, any $\tau$-differentiation at $o$ defines a differentiation, at $o \in M$, of the space of functions of class $C^{\infty}$ on $M$ and hence there exists a vector $v \in V_{o}$ such that $D_{o}-\nabla_{v}$ vanishes on all such functions. If we then apply $D_{o}-\nabla_{v}$ to a vector field $X$ we see easily that $\left(D_{o}-\nabla_{v}\right) X$ depends only on $x$ so that $D_{o}-\nabla_{v}$ defines an endomorphism $a$ on $V_{o}$. Similarly, it defines an endo- 
morphism on $V_{0}^{*}$ which by (3) is simply $-a^{*}$ (where $a^{*}$ designates the transpose of $a$ on $\left.V_{o}^{*}\right)$. Moreover, letting $a_{1}=a$ and $a_{-1}=-a^{*}$ for the present, it follows that $D_{o}-\nabla_{v}$ applied to any tensor field $S$ of type $\gamma=\left(\gamma_{1}, \gamma_{2}, \cdots, \gamma_{k}\right)$ is equal to

$a_{\gamma}=\sum_{i=1}^{k} 1 \otimes 1 \otimes \cdots \otimes a_{\gamma_{i}} \otimes \cdots \otimes 1$ ( $a_{\gamma_{i}}$ the $i$ th term, $k=$ number of terms $)$

applied to $s$. Conversely, given any endomorphism $a$ on $V_{o}$ we may consider the endomorphism $a_{\gamma}$ of $V_{o}$ defined in just this way. Furthermore if $S$ is any tensor field decomposing into the sum $S=\sum_{\gamma} S^{\gamma}$ according to type and we define $\nu(a) S=\sum_{\gamma} a_{\gamma} s^{\gamma}$ we see that $\nu(a)$ is just a $\mathcal{G}$-differentiation at $o$. Thus we may associate to every endomorphism $a$ of $V_{o}$ a $\mathcal{T}$-differentiation $\nu(a)$ at $o$. In the future we shall drop the symbol $\nu$ in $\nu(a)$ and simply understand that any endomorphism $a$ of $V$ by an extension of its domain may be applied to any tensor field in the manner discussed above. It follows then that any $\tau$-differentiation $D_{o}$ at $o \in M$ may be uniquely written as

$$
D_{o}=\nabla_{v}+a
$$

for some vector $v \in V_{o}$ and some endomorphism $a$ of $V_{o}$ and that every such sum is a $\tau$-differentiation. We see then that a $\tau$-differentiation is determined once we know how it acts on functions and vector fields.

.3. If $X$ is a vector field we shall let $\nabla_{\boldsymbol{X}}$ be the derivation of $\mathcal{T}$ defined by setting $\nabla_{X} S$ equal to $\nabla_{x} S$ at any point $o \in M$.

Its action on functions already given, the definition of covariant differentiation on $M$ is uniquely determined in that it satisfies for any two vector fields $X$ and $Y$ the equations

$$
\begin{aligned}
v(X, Y) & =\left(\nabla_{v} X, Y\right)+\left(X, \nabla_{v} Y\right), \\
{[X, Y] } & =\nabla_{X} Y-\nabla_{Y} X
\end{aligned}
$$

where $($,$) designates the inner product in the tangent space at any point$ given by the metric tensor at the point and $[X, Y]$ is just the Poisson bracket of the vector fields $X$ and $Y$.

(1.3.1) expresses the fact that the metric tensor has covariant derivative zero and (1.3.2) the fact that the torsion tensor is zero.

.4. Now if $X$ and $Y$ are any two vector fields, then it is easy to see that

$$
[\nabla x, \nabla Y]-\nabla[x, Y]
$$

defines, at each point $o \in M$, a $\tau$-differentiation. Moreover this $\mathcal{T}$-differentiation vanishes on functions and thus is given by an endomorphism of $V_{o}$. Depending only on $x$ and $y$ this endomorphism, $t(x, y)$, is well known to be the contraction of the curvature tensor, $t$, at $o$ on the last two indices by $x$ and $y$. Letting $T$ be the curvature tensor field and contracting in the same way with $X$ and $Y$ we thus have

$$
\left[\nabla_{X}, \nabla_{Y}\right]-\nabla_{[X, Y]}=T(X, Y)
$$


as a field of tangent space endomorphisms.

Besides being skew-symmetric in $u$ and $v$ the endomorphism $t(u, v)$ satisfies the first Bianchi identity

$$
t(u, v) w+t(v, w) u+t(w, u) v=0 .
$$

.5. We shall call an endomorphism $a$ of $V_{o}$ symmetric (resp. skew-symmetric) if $a$ satisfies $(a u, v)=(u, a v)$ (resp. $(a u, v)=-(u, a v))$ for all $u, v \in V_{o}$, i.e. if the second order covariant tensor obtained from $a$ by lowering its upper index is symmetric (resp. skew-symmetric).

It is well known that $t(u, v)$ is skew-symmetric.

The final relation involving the curvature tensor which we shall make use of in this paper is the second Bianchi identity,

$$
\left[\nabla_{u} T\right](v, w)+\left[\nabla_{v} T\right](w, u)+\left[\nabla_{w} T\right](u, v)=0 .
$$

The contraction again being on the last two indices.

The above identities are proved, for example, in [13].

2. A structure theorem. .1. Let $X$ be any vector field. We may associate to $X$ a field of tangent space endomorphisms $A_{X}$, i.e. a tensor field of one upper index and one lower index, in the following way: For $v \in V_{o}$ we define the value $a_{X}$ of $A_{X}$ at $o$ by

$$
a_{X} v=-\nabla_{v} X .
$$

Consider the derivation $\nabla_{X}+A_{X}$ of $\mathcal{\tau}$. Clearly

$$
\left(\nabla_{X}+A_{X}\right) f=X f
$$

where $f$ is a function.

$$
\begin{aligned}
\left(\nabla_{X}+A_{X}\right) Y & =\nabla_{X} Y+A_{X} Y \\
& =\nabla_{X} Y-\nabla_{Y} X=[X, Y]
\end{aligned}
$$

by (1.3.2).

However if $L_{X}$ is the derivation of $\mathcal{T}$ corresponding to Lie differentiation with respect to $X$, then at each point $L_{X}$ defines a $\mathcal{T}$-differentiation. Moreover,

$$
L_{X} f=X f
$$

for $f$ a function and

$$
L_{X} Y=[X, Y]
$$

for $Y$ a vector field. Thus by $\$ 1.2$

$$
L_{X}=\nabla_{X}+A_{X} .
$$

It is well known that for $X$ and $Y$

$$
\left[L_{X}, L_{Y}\right]=L_{[X, Y]} \text {. }
$$


In fact this follows easily since $\left[L_{X}, L_{Y}\right]-L_{[X, Y]}$ defines a $\tau$-differentiation at each point which vanishes on functions and by the Jacobi identity vanishes on vector fields and hence is zero.

The relation (2.1.3) enables us to give an expression for $A_{[X, Y]}$ in terms of $A_{X}$ and $A_{Y}$. Indeed by (2.1.2) and (2.1.3)

$$
\left[\nabla x+A_{X}, \nabla_{Y}+A_{Y}\right]=\nabla_{[X, Y]}+A_{[X, Y]}
$$

or

$$
\left[\nabla_{X}, \nabla_{Y}\right]+\left[\nabla_{X}, A_{Y}\right]-\left[\nabla_{Y}, A_{X}\right]+\left[A_{X}, A_{Y}\right]=\nabla_{[X, Y]}+A_{[X, Y]} .
$$

But

$$
\begin{aligned}
& {\left[\nabla_{X}, A_{Y}\right]=\nabla_{X}\left(A_{Y}\right),} \\
& {\left[\nabla_{Y}, A_{X}\right]=\nabla_{Y}\left(A_{X}\right)}
\end{aligned}
$$

and since

$$
\left[\nabla_{X}, \nabla_{Y}\right]-\nabla_{[X, Y]}=T(X, Y)
$$

we have

$$
T(X, Y)+\nabla_{X}\left(A_{Y}\right)-\nabla_{Y}\left(A_{X}\right)+\left[A_{X}, A_{Y}\right]=A_{[X, Y]} .
$$

.2. A vector field $X$ is called an infinitesimal motion or Killing vector if the Lie derivative of the metric tensor with respect to $X$ is equal to 0 . This is equivalent to saying that $a_{X}$ is skew-symmetric at all points $o \in M$, or as we shall say $A_{X}$ is skew-symmetric.

Since it is trivial to show that if $A_{X}$ is skew-symmetric then $\nabla_{v}\left(A_{X}\right)$ is likewise skew-symmetric it follows from (2.1.4) that if $X$ and $Y$ are infinitesimal motions then so is $[X, Y]$. Thus if $g$ is the space of all infinitesimal motions on $M, \mathfrak{g}$ becomes a Lie algebra under the Poisson bracket. $\mathfrak{g}$, as is well known, and which will follow later, is finite dimensional; however, finite dimensionality is not understood here to be a requirement for the definition of a Lie algebra.

It is a known fact (see $[13$, p. 45$]$ ) that if $X$ is a vector field then the endomorphism

$$
\nabla_{v}\left(A_{X}\right)-t(x, v)
$$

of $V_{o}$ is the value at $o$ of the Lie derivative, by $X$, of the affine connection contracted by $v \in V_{o}$. Since if $X \in \mathfrak{g}$ the Lie derivative of the affine connection is zero it follows of course that for $X \in g$ the above expression vanishes.

For completeness we shall give a proof of this which does not involve a computation of the Lie derivative of the affine connection. We state it in the following form.

Lemma 2.2. Let $X$ be a vector field. Then at any point $o \in M, \nabla_{v}\left(A_{X}\right)$ 
$=t(x, v)$ for all $v \in V_{0}$ if and only if $\nabla_{v}\left(A_{X}\right)$ is skew-symmetric for all $v \in V_{0}$.

Proof. It is of course trivial that if the equality holds then $\nabla_{v}\left(A_{X}\right)$ is skew-symmetric for all $v \in V_{o}$.

Let $X$ be arbitrary. We first show that the vector

$$
\nabla_{\boldsymbol{y}}\left(A_{X}\right) z-t(x, y) z
$$

as a bilinear function of $y$ and $z$ is symmetric in $y$ and $z$. In fact embed $y$ and $z$ in the respective vector fields $Y$ and $Z$.

Now

$$
\begin{aligned}
\nabla_{Y}\left(A_{X}\right) Z & =\left[\nabla_{Y}, A_{X}\right] Z \\
& =\nabla_{Y} A_{X} Z-A_{X} \nabla_{Y} Z=-\nabla_{Y} \nabla_{Z} X-A_{X} \nabla_{Y} Z .
\end{aligned}
$$

Then

But by (1.4.2)

$$
\begin{aligned}
\nabla_{Y}\left(A_{X}\right) Z-\nabla_{Z}\left(A_{X}\right) Y & =\left[\nabla_{Z}, \nabla_{Y}\right] X+A_{X}[Z, Y] \\
& =\left(\left[\nabla_{\boldsymbol{Z}}, \nabla_{Y}\right]-\nabla_{[Z, Y]}\right) X \\
& =T(Z, Y) X
\end{aligned}
$$

$$
t(z, y) x=t(x, y) z-t(x, z) y .
$$

Thus we have the symmetry in $y$ and $z$ expressed in the relation

$$
\nabla_{y}\left(A_{x}\right) z-t(x, y) z=\nabla_{z}\left(A_{x}\right) y-t(x, z) y .
$$

Let

$$
\phi(v, u, w)=\left(\nabla_{v}\left(A_{X}\right) u, w\right)-(t(x, v) u, w) .
$$

Then $\phi$ is symmetric in $u$ and $v$. If we now make the assumption that $\nabla_{v}\left(A_{X}\right)$ is skew-symmetric for all $v \in V_{o}$, then $\phi(v, u, w)$ is skew-symmetric in $u$ and $w$. But any trilinear form symmetric in two variables and skewsymmetric in two others is necessarily identically zero. Thus

$$
\nabla_{v}\left(A_{X}\right)=t(x, v) \text {. }
$$

.3. If we consider the question as to how much information is yielded about $X \in \mathfrak{g}$ by knowledge of $x$ and $a_{X}$, we are led, heuristically at any rate, to thinking of $X$ decomposing into a sum of an infinitesimal translation at $o$ in the direction of $x$ and an infinitesimal rotation about $o$ given in the tangent space of $o$ by $a_{X}$. Indeed if we consider for the moment the case where $M$ is Euclidean $n$-space with the usual metric, and we let $o$ be the origin, we know that $\mathfrak{g}$ decomposes into the direct sum $\mathfrak{g}=\mathfrak{a}+V$ where $\mathfrak{a}$ is the subalgebra of $\mathfrak{g}$ composed of all elements in $\mathfrak{g}$ vanishing at $o$ and $V$ is the commutative subalgebra of all infinitesimal translations. Now if we let $\mathfrak{a}_{o}$ be the Lie algebra of all skew-symmetric endomorphisms of $V_{0}$ and we define $\mathrm{g}_{o}=\mathfrak{a}_{o}+V_{o}$ and introduce a bracket operation into $\mathrm{g}_{o}$ by setting 


$$
\begin{gathered}
{\left[a_{1}, a_{2}\right]=a_{1} a_{2}-a_{2} a_{1} \in \mathfrak{a}_{o},[a, v]=a(v) \in V_{o},} \\
{[v, a]=-a(v) \in V_{o},\left[v_{1}, v_{2}\right]=0}
\end{gathered}
$$

for $a, a_{1}, a_{2} \in \mathfrak{a}_{o} ; v, v_{1}, v_{2} \in V_{o}$, then $\mathrm{g}_{o}$ is a Lie algebra and $\mathfrak{g}$ is isomorphic to $\mathrm{g}_{o}$ under the mapping which sends $X$ into $a_{X}+x$. This mapping of course sends $\mathfrak{a}$ into $\mathfrak{a}_{o}$ and $V$ into $V_{o}$.

More generally it was shown by $\mathrm{E}$. Cartan that if $M$ is a simply connected Riemannian symmetric space and $o$ is any point of $M$, then $g$ is isomorphic to $\mathfrak{g}_{o}=\mathfrak{f}_{o}+V_{o}$ where $\mathfrak{l}_{o}$ is the subalgebra of all skew-symmetric endomorphisms $a$ of $V_{o}$ which leave invariant the curvature tensor at $o \in M$ (infinitesimally, i.e., $a T=0)$. The bracket relation in $g_{o}$ is introduced in the same way as before with respect to $\left[\mathfrak{t}_{o}, \mathfrak{l}_{o}\right],\left[\mathfrak{t}_{o}, V_{o}\right],\left[V_{o}, \mathfrak{t}_{o}\right]$, but for $\left[V_{o}, V_{o}\right]$ we now have more generally $\left[V_{o}, V_{o}\right] \subseteq \mathfrak{l}_{o}$ where

$$
\left[v_{1}, v_{2}\right]=t\left(v_{2}, v_{1}\right) \quad \text { for } v_{1}, v_{2} \in V_{o} .
$$

The isomorphism between $\mathfrak{g}$ and $\mathfrak{g}_{o}$ is again established by mapping $X$ into $a_{X}+x$.

We return now to the case where $M$ is perfectly general. Let $\mathfrak{a}_{o}$ be all skew-symmetric endomorphisms of $V_{o}$. Let $\widehat{\mathrm{g}}_{0}=\mathfrak{a}_{o}+V_{o}$. We introduce into $\widehat{\mathrm{g}}_{0}$ a bracket operation where

$$
\begin{aligned}
{\left[a_{1}, a_{2}\right] } & =a_{1} a_{2}-a_{2} a_{1},[a, v]=a(v), \\
{[v, a] } & =-a(v),\left[v_{1}, v_{2}\right]=t\left(v_{2}, v_{1}\right)
\end{aligned}
$$

for $a, a_{1}, a_{2} \in \mathfrak{a}_{o}$ and $v, v_{1}, v_{2} \in V_{o} . \hat{\mathfrak{g}}_{o}$ is not in general a Lie algebra under this bracket (the Jacobi identity fails in the general case) but of course subalgebras of $\hat{\mathfrak{g}}_{o}$ may be Lie algebras. We have seen that in the symmetric case $\mathfrak{g}$ is isomorphic to a subalgebra $\mathrm{g}_{o}$ of $\hat{\mathrm{g}}_{o}$ under the mapping $X \rightarrow a_{X}+x$. The following theorem states that this is true in general.

THEOREM 2.3. Let $o \in M$. Let $\widehat{\mathrm{g}}_{o}=\mathfrak{a}_{o}+V_{o}$ be the algebra defined in $\S 2.3$. Let $\mathfrak{g}$ be the Lie algebra of infinitesimal motions on $M$. Let $\theta_{o}: \mathfrak{g} \rightarrow \hat{\mathfrak{g}}_{o}$ be the mapping defined by

$$
\theta_{o}(X)=X_{o}=a_{X}+x
$$

and $\mathfrak{g}_{o}=\theta_{o}(\mathfrak{g})$; then $\theta_{o}$ is an isomorphism of $\mathfrak{g}$ onto $\mathfrak{g}_{o}$.

Proof. Implicit in the statement of the theorem is that, first of all, $\theta_{0}$ is one-one. This is equivalent to the statement that an infinitesimal motion $X$ is uniquely determined over all of $M$ by the value of $A_{X}$ and $X$ at any one point. To see this, let $M$ and let $r \rightarrow o(r)$ be a differentiable curve, $1 \leqq r \leqq 2$, connecting $o_{1}$ with $o_{2}$. Then if $a_{X}(r)$ and $x(r)$ are respectively the values of $A_{X}$ and $X$ respectively at $o(r)$, and $v(r)$ is the tangent vector to the curve at $o(r)$, then it follows from (2.1.1) and Lemma 2.2 that the pair $x(r)$ and $a_{X}(r)$ satisfy the linear differential equations 


$$
\begin{aligned}
\nabla_{v(r)} x(r) & =-a_{X}(r) v(r), \\
\nabla_{v(r)} a_{X}(r) & =t(x(r), v(r))
\end{aligned}
$$

so that a knowledge of them at any one point of the curve determines their value at any other.

To show $\theta_{0}$ is a homomorphism, let $X, Y \in \mathfrak{g}$ and let $X_{0}=\theta_{0} X, Y_{0}=\theta_{0} Y$. Now by (2.1.4)

$$
T(X, Y)+\nabla_{X}\left(A_{Y}\right)-\nabla_{Y}\left(A_{X}\right)+\left[A_{X}, A_{Y}\right]=A_{[X, Y]} .
$$

But since $X$ and $Y$ are infinitesimal motions we have by Lemma 2.2 that

$$
\begin{aligned}
& \nabla_{X}\left(A_{Y}\right)=T(Y, X), \\
& \nabla_{Y}\left(A_{X}\right)=T(X, Y) .
\end{aligned}
$$

Thus

$$
A_{[X, Y]}=T(Y, X)+\left[A_{X}, A_{Y}\right] .
$$

Moreover by (1.3.2)

$$
[X, Y]=\nabla_{X} Y-\nabla_{Y} X
$$

or by $(2.1 .1)$

$$
[X, Y]=A_{X} Y-A_{Y} X .
$$

Hence, by evaluating $[X, Y]$ and $A_{[X, Y]}$ at $o$ we see that

$$
\theta_{o}[X, Y]=\left[X_{o}, Y_{o}\right] \text {. }
$$

Note. With respect to the curve $r \rightarrow o(r)$ mentioned in the proof of Theorem 2.3 and by means of the linear differential equations (2.3.1) and (2.3.2) we obtain, by solving these equations, a linear one-one mapping from $\widehat{\mathrm{g}}_{o_{1}}$ to $\hat{g}_{o_{2}}$. If now $o_{1}=o_{2}=o$ it is clear that the elements of $g_{o}$ are pointwise fixed. In fact it is not hard to see that $g_{o}$ is characterized as the set of those elements in $\hat{\mathrm{g}}_{o}$ which remain invariant under all mappings of $\hat{\mathrm{g}}_{o}$ into itself obtained in this way for all closed differentiable curves passing through $o$.

For any $X_{o} \in \widehat{\mathfrak{g}}_{o}, X_{o}=a+x$, we define $l_{X_{o}}$ to be the $\mathcal{T}$-differentiation $\nabla_{x}+a$ at $o$. The question as to whether the Jacobi identity is satisfied for three elements $X_{o}, Y_{o}$ and $Z_{o} \in \hat{\mathfrak{g}}_{o}$ is, by using the second Bianchi identity (1.5.1), seen to be equivalent to the question as to whether

$$
\left(l_{X_{0}} T\right)(y, z)+\left(l_{Y_{o}} T\right)(z, x)+\left(l_{z_{o}} T\right)(x, y)
$$

vanishes. (The contraction is again on the last two indices.) In case $X_{o}=\theta_{o} X$ for some vector field $X$, then $l_{X_{0}}$ is by (2.1.2) just the Lie derivative $L_{X}$ evaluated at $o$. If therefore $X \in \mathfrak{g}$ it follows that $l_{X_{0}} T=0$ since the curvature tensor is invariant under motions.

3. Holonomy and infinitesimal motions. .1. Let $\psi_{0}$ be the homogeneous holonomy group at $o \in M$. $\psi_{o}$ is a group of orthogonal endomorphisms of the 
tangent space $V_{o}$, orthogonal with respect to the inner product in $V_{o}$. We recall, by definition, that an orthogonal endomorphism $\alpha$ belongs to $\psi_{o}$ if the endomorphism, $\alpha: V_{o} \rightarrow V_{o}$, is induced by transporting (by parallelism) $V_{o}$ completely around a closed piecewise differentiable curve on $M$. It returns to its initial position only after being subjected to the rotation $\alpha$.

The restricted homogeneous holonomy group $\sigma_{o}$ at $o$ is a normal subgroup of $\psi_{o}$ and is obtained in the same way as $\psi_{0}$ except that the curves are restricted to be homotopic to zero. It is a known result [3] that $\sigma_{o}$ as a group of orthogonal endomorphisms is compact and connected and is in fact the connected component of the identity in $\psi_{0}$.

.2. Let $\dot{z}_{o}$ be the Lie algebra of $\sigma_{o} . \dot{z}_{o}$ is of course a subalgebra of $\mathfrak{a}_{o}$. Consider, in $\mathfrak{a}_{o}$, the inner product,

$$
B\left(a_{1}, a_{2}\right)=\text { trace } a_{1} a_{2} .
$$

$B$ is clearly negative definite. Let $\mathfrak{u}_{o}$ be the orthocomplement of $\boldsymbol{z}_{o}$ in $\mathfrak{a}_{o}$ with respect to $B$.

Let $\mathfrak{f}^{\circ}$ be the set of $X \in \mathfrak{g}$ such that $x$ vanishes and let $\mathfrak{f}_{o}=\theta_{o} \mathfrak{f}^{\circ} \mathfrak{f}^{\circ}$ is a subalgebra of $\mathfrak{g}$; in fact $\mathfrak{f}_{o}$ is a subalgebra of $\mathfrak{a}_{o}$. If $K^{o}$ is the group of all motions of $M$ which keep $o$ fixed then its Lie algebra, represented as vector fields on $M$, is a subalgebra of $\mathfrak{f}^{\circ}$ so that it is natural to call $\mathfrak{t}^{\circ}$ the isotropy algebra at $o$ and $\mathfrak{f}_{o}$ the linear isotropy algebra at $\boldsymbol{o}$.

It is a simple fact that if $a \in \mathfrak{l}_{o}$ then $a$ lies in the normalizer of $8_{o}$. It is not much harder to show this is true for any $a_{X}$ where $X \in \mathfrak{g}$. A more general statement is derived as an easy consequence of the next lemma; it is more general in that it deals with $\psi_{o}$ instead of $\sigma_{o}$ or $\boldsymbol{B}_{0}$.

For any $X \in \mathfrak{g}$ we consider the decomposition of $a_{X}$,

$$
a_{X}=b_{X}+e_{X},
$$

where $b_{X} \in B_{o}$ and $e_{X} \in \mathfrak{u}_{o}$. Let $E_{X}$ (resp. $B_{X}$ ) be the field of tangent space endomorphisms which at any point $o$ takes the value $e_{X}$ (resp. $b_{X}$ ).

\section{Lemma 3.2. The field $E_{X}$ is covariant constant.}

Proof. We use in the proof two basic facts about holonomy; (1) if $o(r)$, $r_{1} \leqq r \leqq r_{2}$, is a differentiable curve then $\boldsymbol{B}_{o\left(r_{1}\right)}$ as a subspace of $\mathfrak{a}_{o\left(r_{1}\right)}$ is carried into $B_{o\left(r_{2}\right)}$ under parallel transport along the curve, and (2) at any point $o$ and for any $u, v \in V_{o}, t(u, v) \in \varepsilon_{o}$, see, for example, [1].

But from (1) it follows that the orthocomplement $\mathfrak{u}_{o\left(r_{1}\right)}$ of $\boldsymbol{B}_{o\left(r_{1}\right)}$ is carried into $\mathfrak{u}_{o\left(r_{2}\right)}$ under parallel transport along the curve. Thus to prove the lemma it is sufficient to show the covariant derivative of $A_{x}$ by any vector $v \in V_{o}$ lies in $\boldsymbol{B}_{0}$. Now by Lemma 2.2 ,

$$
\nabla_{v}\left(A_{X}\right)=t(x, v) \in 8_{0} .
$$

Thus we have shown $\nabla_{v}\left(E_{X}\right)=0$. Q.E.D. 
.3. We are interested in the question as to when $a_{X} \in z_{0}$, i.e. when $e_{X}=0$, or equivalently, $E_{X}=0$.

It follows from a result of Lichnerowicz [12] that this is the case when the Ricci tensor is not zero and $\varepsilon_{0}$ acts irreducibly on $V_{o}$. In fact that author uses his result to prove that $a_{X} \in \boldsymbol{B}_{o}$ when $X \in \mathfrak{l}^{\circ}$ under those assumptions. For completeness we repeat his argument (slightly modified) here. Assume $E_{X} \neq 0$. Since $\dot{B}_{o}$ acts irreducibly it follows that $M$ is pseudo-Kahlerian. The result in [12] alluded to above is that if $M$ is pseudo-Kahlerian and the Ricci tensor is not zero then $\boldsymbol{B}_{o}$ has a nonzero center. But if $c \in \boldsymbol{B}_{o}$ is in the center then $c, e_{X}$, and the identity endomorphism generate a three-dimensional commutative algebra in the centralizer of $\boldsymbol{B}_{0}$. Since the latter acts irreducibly this contradicts Schur's lemma.

Of course we know that in general $a_{X} \notin B_{o}$, as Euclidean space clearly illustrates. We shall show, however, that $a_{X} \in z_{0}$ whenever $M$ is compact.

Theorem 3.3. Let $M$ be a compact Riemannian manifold, $X$ an infinitesimal motion on $M$, and $a_{X}$ the endomorphism of the tangent space $V_{o}$ at an arbitrary point $o \in M$ defined by $a_{X} v=-\nabla_{v} X$ for any $v \in V_{o}$. Then $a_{X} \in 8_{o}$, the Lie algebra of the restricted homogeneous holonomy group at $o$.

Proof. We use the notation of $\S 3.2$. We shall show $E_{X}=0$. Let $Y$ be a vector field defined by

$$
Y=E_{X} X .
$$

That is, $E_{X}$ is applied to $X$ at every point. For any $v \in V_{o}$

$$
\nabla_{v} Y=E_{X} \nabla_{v} X
$$

(by Lemma 3.2).

Thus

$$
a_{Y} v=e_{X} a_{X} v
$$

and consequently

$$
A_{Y}=E_{X} A_{X} .
$$

Assume for the present that $M$ is orientable. It is just the statement of Green's theorem, for example see [13, p. 31] that if $Z$ is any vector field on $M$ then

$$
\int \operatorname{tr} A_{Z} d v=0
$$

where $d v$ is the volume element on $M$ associated with the metric.

Thus

$$
\int \operatorname{tr} A_{Y} d v=\int \operatorname{tr}\left[E_{X}^{2}+E_{X} B_{X}\right] d v=0 .
$$

But $\operatorname{tr} E_{X} B_{X}=0$ at every point $o$ by definition of $\mathfrak{u}_{o}$. Thus 


$$
\int \operatorname{tr} E_{X}^{2} d v=0
$$

However $\operatorname{tr} E_{X}^{2}$ is constant on $M$ since $E_{X}$ is covariant constant. Thus

$$
\operatorname{tr} \stackrel{2}{e}_{X}^{2}=B\left(e_{X}, e_{X}\right)=0 .
$$

But since $B$ is negative definite, $e_{X}=0$.

In case $M$ is not orientable then $M$ has a two sheeted compact locally isometric covering space $M$ which is orientable. The vector field $X$ when lifted to $M$ determines an infinitesmal motion $\widehat{X}$. The above argument shows that $e_{\widehat{X}}=0$ and hence obviously $e_{X}=0$. Q.E.D.

4. Transitivity. .1. Let $\mathrm{g}^{*}$ be a subalgebra of $\mathfrak{g}$. Let $V\left(\mathrm{~g}_{0}^{*}\right)$ be the subspace of $V_{0}$ defined by

$$
V\left(\mathfrak{g}_{0}^{*}\right)=\left\{x \in V_{o} \mid X \in \mathfrak{g}^{*}\right\} .
$$

We shall say that $\mathrm{g}^{*}$ is transitive on $M$ if $V\left(\mathrm{~g}_{o}^{*}\right)=V_{o}$ for every $o \in M$.

.2. Let $G$ be a connected Lie group and $H$ a compact subgroup. Let $M$ be the homogeneous space $G / H$ (left coset space) when the latter is provided with a metric tensor which is kept invariant by the action of $G$ on $G / H$. In this case we see that $\mathfrak{g}$ contains a subalgebra $\mathfrak{g}^{*}$ which is transitive on $M$. In fact the right invariant Lie algebra of $G$ maps homomorphically into $g$ under the mapping which associates to every one-parameter group $g(t)$ of $G$ the vector field $X \in \mathfrak{g}$ on $M$ where

$$
X f(o)=\lim _{t \rightarrow 0} \frac{f(g(t) o)-f(o)}{t}
$$

for any $C^{\infty}$ function, $f(o)$, on $M$. The image, $\mathrm{g}^{*}$, under this mapping is transitive on $M$.

More generally then if $M$ is an open submanifold of such a homogeneous space, then $\mathfrak{g}$ contains a subalgebra which is transitive on $M$.

.3. For any point $o \in M$ consider the subalgebra $\mathfrak{f}^{\circ} \subseteq \mathfrak{g}$, see $\S 3.2$. It is not hard to see that $\mathfrak{l}^{\circ}$ is reductive in $\mathfrak{g}$ (the adjoint representation of $\mathfrak{f}^{\circ}$ on $\mathfrak{g}$ is completely reducible). In fact $\mathfrak{g}$ is isomorphic to $\mathfrak{g}_{o} \subseteq \mathfrak{a}_{o}+V_{o}$ and $\mathfrak{f}^{\circ}$ is isomorphic to $\mathfrak{f}_{o} \subseteq \mathfrak{a}_{o}$ (Theorem 2.3). Let

$$
\mathfrak{l}_{o}=\left\{a_{X} \mid X \in \mathfrak{g}\right\} .
$$

Of course $\mathfrak{l}_{o} \subseteq \mathfrak{l}_{o} \subseteq \mathfrak{a}_{o}$. $\mathfrak{a}_{o}$ is a Lie algebra under the usual bracket operation for endomorphisms. Furthermore any subalgebra, as for example $\mathfrak{f}_{o}$, is reductive in $\mathfrak{a}_{o}$. Moreover, by Theorem $2.3, \mathfrak{l}_{o}$ is invariant under the adjoint representation of $\mathfrak{f}_{o}$ on $\mathfrak{a}_{o}$. Let $\dot{\mathfrak{j}}_{o}$ be the orthogonal complement (:vith respect to the bilinear form $B$ ) of $\mathfrak{t}_{o}$ in $\mathfrak{l}_{o}$. Thus

$$
\mathfrak{l}_{o}=\mathfrak{l}_{o}+\mathfrak{i}_{o}, \quad \mathfrak{l}_{o} \cap \mathfrak{l}_{o}=0
$$


where $\left[\mathfrak{t}_{o}, \mathfrak{t}_{o}\right] \subseteq \mathfrak{l}_{o},\left[\mathfrak{t}_{o}, \dot{\mathfrak{t}}_{o}\right] \subseteq \mathfrak{i}_{o}$.

Define

$$
\mathfrak{p}_{o}=\left\{X_{o} \in \mathfrak{g}_{0} \mid a_{X} \in \dot{\mathfrak{j}}_{0}\right\} .
$$

Then it follows that

$$
\mathfrak{g}_{o}=\mathfrak{l}_{o}+\mathfrak{p}_{o}, \quad \mathfrak{l}_{o} \cap \mathfrak{p}_{o}=0
$$

where $\left[\mathfrak{f}_{o}, \mathfrak{p}_{o}\right] \subseteq \mathfrak{p}_{o}$.

.4. The mapping of $\mathfrak{p}_{o}$ into $V\left(\mathfrak{g}_{o}\right)$ which sends $X_{o}$ into $x$ is one-one onto and defines an equivalence between the representation of $\mathfrak{t}_{o}$ on $\mathfrak{p}_{o}$ and that on $V\left(\mathfrak{g}_{o}\right)\left(V\left(\mathfrak{g}_{o}\right)\right.$ is an invariant subspace of $V_{o}$ under the action of $\mathfrak{t}_{o}$ as endomorphisms). Since the latter is reductive the action of $\mathfrak{f}_{o}$ in $\mathfrak{p}_{o}$ is reductive and hence the adjoint representation of $\mathfrak{t}_{o}$ on $\mathfrak{g}_{o}$ is reductive.

Now if $M$ is a simply connected Riemannian symmetric space then it is a known result of $\mathrm{E}$. Cartan that $a_{X}=0$ if $\theta_{o} X=X_{o} \in \mathfrak{p}_{o}$, that is, $\dot{t}_{o}=0$ or $\mathfrak{l}_{o}=\mathfrak{l}_{o}$. Such a vector field is an infinitesimal transvection at $o \in M$. It is also known that $\mathrm{g}$ is transitive on $M$. Conversely, if we assume that $\mathrm{g}$ is transitive on $M$ and that $\dot{\mathrm{t}}_{o}=\mathfrak{f}_{o}$ for all $o \in M$, then $M$ must be Riemannian symmetric since if $X \in \mathrm{g}$ then we know that $L_{X} T=0$, i.e. "the curvature tensor is invariant under motions." Thus since $L_{X}=\nabla x+A_{X}$

$$
\nabla_{x} T=-a_{X} T .
$$

However, since $V\left(g_{o}\right)=V_{o}$ and $a_{X}=0$ when $\theta_{o} X \in \mathfrak{p}_{o}$ it follows that $\nabla_{v} T=0$ for all $v \in V_{o}$. Thus the curvature tensor is covariant constant which is another way of saying that $M$ is symmetric. More generally we see that $M$ is symmetric if $g$ is transitive and $a_{X} T=0$ for all $X \in \mathfrak{g}$ and all $o \in M$. Thus a distinction between a symmetric space and a nonsymmetric space on which $\mathrm{g}$ is transitive is that in the latter case there always exists $X$ for which $a_{X} T \neq 0$ whereas in the former $a_{X} T=0$ for all $X \in \mathrm{g}$.

Now if $M$ is symmetric and simply connected it is a result of $\mathrm{E}$. Cartan that

$$
\mathfrak{B}_{o} \subseteq \mathfrak{l}_{o}\left(=\mathfrak{l}_{o}\right) \subseteq n\left(\mathbb{8}_{o}\right)
$$

where $n\left(B_{o}\right)$ is the normalizer of $\dot{B}_{o}$.

Now for the general case let $\mathfrak{h}_{o}$ be the Lie subalgebra of $\mathfrak{a}_{o}$ generated by $\mathfrak{l}_{o}$ (in the case above $\mathfrak{h}_{o}=\mathfrak{l}_{o}$ since $\mathfrak{l}_{o}$ is already a Lie algebra). The next theorem extends the result expressed by (4.4.2) to the case where $\mathfrak{g}$ is only assumed transitive on $M$ and $\mathfrak{h}_{o}$ replaces $\mathfrak{l}_{o}$.

In fact it is not necessary to deal with the full Lie algebra of infinitesimal motions and in fact Corollary 4.5 illustrates why the following theorem should be formulated in terms of a transitive subalgebra of $\mathfrak{g}$.

Theorem 4.4. Assume $\mathrm{g}^{*}$ is a Lie algebra of infinitesimal motions on $M$ which is transitive (see \$4.1.). For any point $o \in M$ let $\mathfrak{h}_{0}^{*}$ be the Lie algebra of 
skew-symmetric endomorphisms of $V_{0}$ generated by all $a_{X}$ for $X \in \mathfrak{g}^{*}$. Then if $\mathbb{B}_{o}$ is the Lie algebra of the restricted holonomy group,

$$
\mathrm{B}_{0} \subseteq \mathfrak{h}_{0}^{*} \subseteq n\left(B_{0}\right)
$$

where $n\left(B_{0}\right)$ is the normalizer of $\dot{B}_{0}$.

Proof. It was already mentioned in $\$ 3.2$ that $a_{X} \in n\left(8_{0}\right)$ for any $X \in \mathfrak{g}$, a fact which follows from Lemma 3.2 since the latter implies $\left[z_{o}, e_{X}\right]=0$. Thus $\mathfrak{h}_{0}^{*} \subseteq n\left(\xi_{o}\right)$. In order to prove $\boldsymbol{\Xi}_{o} \subseteq \mathfrak{h}_{o}^{*}$ we first need a lemma.

Lemma 4.4. Let $S$ be a tensor field in an arbitrary Riemannian manifold $M$ which is invariant under motions, i.e., $L_{X} S=0$ for all $X \in \mathfrak{g}$.

Let $W_{o}$ be any subspace of $\tau\left(V_{o}\right)$ which is invariant under $a_{X}$ for all $X \in \mathfrak{g}$ and such that $s \in W_{o}$. Then for any $X_{1}, X_{2}, \cdots, X_{k} \in \mathfrak{g}$ the value of the field

$$
\nabla x_{1} \nabla_{x_{2}} \cdots \nabla_{x_{k}} S
$$

at $o \in M$ is contained in $W_{o}$.

Proof of Lemma 4.4. If $Y \in \mathfrak{g}$ and $X$ is arbitrary then it follows easily from (2.1.2) and Lemma 2.2 that as operators on $\mathcal{T}$

$$
\left[L_{Y}, \nabla_{X}\right]=\nabla_{[Y, X]}
$$

and consequently for any $X_{2}, \cdots, X_{k}$

Thus

$$
\left[L_{Y}, \nabla x_{2} \cdots \nabla_{X_{k}}\right]=\sum_{i=2}^{k} \nabla_{X_{2}} \cdots \nabla_{\left[Y, X_{i}\right]} \cdots \nabla_{X_{k}} .
$$

(4.4.3) $L_{Y} \nabla x_{2} \cdots \nabla x_{k}=\nabla x_{2} \cdots \nabla x_{k} L_{Y}+\sum_{i=2}^{k} \nabla x_{2} \cdots \nabla\left[Y, X_{i}\right] \cdots \nabla x_{k}$.

Now assume $Y=X_{1}$ and $X_{1}, X_{2} \cdots X_{k} \in \mathfrak{g}_{o}$. Assume also that the lemma is true for $k-1$. If then we apply (4.4.3) to $S$ we see that $L_{X_{1}} \nabla x_{2} \cdots \nabla x_{k} S$ evaluated at $o$ is contained in $W_{o}$. But $L_{X_{1}}=\nabla_{X_{1}}+A_{X_{1}}$. Thus

$$
\nabla x_{1} \nabla_{x_{2}} \cdots \nabla_{x_{k}} S=L_{x_{1}} \nabla_{x_{2}} \cdots \nabla_{x_{k}} S-A_{x_{1}} \nabla_{x_{2}} \cdots \nabla_{x_{k}} S .
$$

But $\nabla_{X_{2}} \cdots \nabla_{x_{k}} S$ at $o$ lies in $W_{o}$ and since $W_{o}$ is invariant under $a_{X_{1}}$ we see that $\nabla x_{1} \nabla_{x_{2}} \cdots \nabla_{x_{k}} S$ at $o \in M$ lies also in $W_{o}$. But we already know that $s \in W_{\text {o. }}$ Q.E.D.

Proof of Theorem 4.4 continued. Assume $\mathrm{g}^{*}$ is transitive on $M$. We apply Lemma 4.4 in the case where $S=T$, the curvature tensor. Let $W_{o}$ be the subspace $V_{o} \otimes V_{o}^{*} \otimes V_{o}^{*} \otimes V_{o}^{*}$ consisting of all $s$ such that for all $u, v \in V_{o}$,

$$
s(u, v) \in \mathfrak{h}_{0}^{*}
$$

where $s(u, v)$ means the contraction of $s$ on the last two indices by the vectors $u, v$. 
To show that $W_{o}$ is invariant under $a_{X}$ we observe that for any $s \in V_{0}$ $\otimes V_{o}^{*} \otimes V_{o}^{*} \otimes V_{o}^{*}$ and any $u, v \in V_{o}$

$$
\left(a_{X} s\right)(u, v)=\left[a_{X}, s(u, v)\right]-s\left(a_{X} u, v\right)-s\left(u, a_{X} v\right)
$$

so that if $s \in W_{o}$ it follows that $a_{X} s \in W_{0}$.

Thus to show that

$$
\left(\nabla_{x_{1}} \nabla x_{2} \cdots \nabla_{x_{k}} T\right)(u, v)
$$

lies in $\mathfrak{h}_{o}^{*}$ for all $X_{1}, X_{2} \cdots X_{k} \in \mathfrak{g}$ it follows from Lemma 4.4 that all we have to show is that $t(u, v) \in \mathfrak{h}_{o}^{*}$ for all $u, v \in V_{o}$. It is at this point that we first use transitivity. Given any $u, v \in V_{o}$, by the transitivity of $\mathfrak{g}^{*}$, there exists $X, Y \in \mathfrak{g}^{*}$ such that $x=u, y=v$. Now by (2.3.3)

$$
\left[A_{X}, A_{Y}\right]-A_{[X, Y]}=T(X, Y) .
$$

Thus $t(u, v) \in \mathfrak{h}_{o}^{*}$ for all $u, v \in V_{o}$.

We assert it follows from a theorem of Chevalley and Nijenhuis, see [8], that the Lie algebra generated by all endomorphisms of $V_{o}$ of the form

$$
\left(\nabla_{x_{1}} \nabla_{x_{2}} \cdots \nabla_{x_{k}} T\right)(u, v)
$$

where $X_{i} \in \mathfrak{g}, u, v \in V_{o}$, is $\dot{s}_{o}$.

In fact if we form this algebra at each point $o \in M$ where the vector fields $X_{i}$ are arbitrary then it follows from that theorem that if the dimension of the algebra is constant over $M$ then at each point $o \in M$ the algebra is $\mathbb{B}_{0}$. What is asserted here is that since $g^{*}$ is transitive, we need only consider vector fields belonging to $\mathrm{g}^{*}$ and that the dimension of the resulting algebra is constant. The first of these assertions follows from the fact that if $X_{1}, X_{2}, \cdots$, $X_{n} \in \mathrm{g}^{*}$ is such that $x_{1}, x_{2}, \cdots, x_{n}$ form a basis of $V_{o}$, then any vector field $Y$ equals $\sum_{i-1}^{n} f_{i} X_{i}$ in a neighborhood of $o$ where $f_{i}, i=1,2, \cdots, n$, are suitable chosen functions. The second follows from the fact that the transitivity of $\mathrm{g}^{*}$ yields the existence of two neighborhoods $V_{o}$ and $V_{o}^{\prime}$ of $o$ such that for each $p \in V_{0}^{\prime}$ there exists an isometric mapping of $V_{0}$ onto a neighborhood of $p$ in which $o$ maps into $p$. Consequently the dimension of the algebra is constant over $M$. Q.E.D.

.5. As mentioned before if the Ricci tensor of $M$ does not vanish and $\dot{z}_{0}$ acts irreducibly (i.e. $M$ is irreducible) then $a_{X} \in \Xi_{o}$ for all $X \in \mathfrak{g}$, or as proved before (Theorem 3.3) this conclusion holds if $M$ is compact. Consequently, we have, combining these facts with Theorem 4.4,

Theorem 4.5. Let $M$ be a Riemannian manifold and $\mathfrak{g}^{*}$ any Lie algebra of infinitesimal motions which is transitive on $M$ (see \$4.1). Now if either one of the following conditions hold:

(a) $M$ is compact

or

(b) $M$ is irreducible and the Ricci tensor does not vanish, then 


$$
\mathfrak{h}_{0}^{*}=\mathfrak{g}_{0}
$$

where $B_{0}$ is the Lie algebra of the restricted homogeneous holonomy group at $o \in M$ and $\mathfrak{h}_{0}^{*}$ is the Lie algebra of endomorphisms of $V_{o}$ generated by all $a_{X}$ (see § 2.1) for $X \in \mathfrak{g}^{*}$.

CoROllary 4.5. Let $G$ be any connected Lie group and $K$ a compact subgroup. Let $M$ be the homogeneous space $G / K$ (left coset space) when the latter is provided with a metric tensor which is kept invariant by the action of $G$ on $G / K$.

Now if either one of the following conditions holds:

(1) $G$ is compact

or

(2) $M$ is irreducible and the Ricci tensor does not vanish, then

$$
\mathfrak{h}_{0}^{*}=\mathfrak{g}_{0}
$$

where $\mathfrak{h}_{0}^{*}$ is the Lie algebra of endomorphisms of $V_{o}$ generated by all $a_{X}$ where $X$ runs through the Lie algebra of infinitesimal motions on $M$ arising from the Lie algebra of $G$ (see \$4.2) and $\dot{B}_{0}$ is the Lie algebra of the restricted homogeneous holonomy group at 0 .

\section{REFERENCES}

1. W. Ambrose and I. M. Singer, $A$ theorem on holonomy, Trans. Amer. Math. Soc. vol. 75 (1953) pp. 428-443.

2. A. Borel and A. Lichnerowicz, Espaces riemannien et hermitiens symetrique, C. R. Acad. Sci. Paris vol. 234 (1952) pp. 2332-2334.

3. - Groupes d'holonomie des varietes riemanniennes, C. R. Acad. Sci. Paris vol. 234 (1952) pp. 1835-1837.

4. E. Cartan, Sur une classe remarquable d'espaces de Riemann, Bull. Soc. Math. France vol. 54 (1926) pp. 214-264; vol. 55 (1927) pp. 114-134.

5. — La géométrie des groupes de transformations, J. Math. Pures Appl. vol. 6 (1927) pp. 1-119.

6. R. Hermann, Sur les isometries infinitesimales et le group d'holonomie d'un espace de Riemann, C. R. Acad. Sci. Paris vol. 239 (1954) pp. 1178-1180.

7. - Sur les automorphismes infinitesimaux d'une G-structure, C. R. Acad. Sci. Paris vol. 239 (1954) pp. 1760-1761.

8. A. Nijenhuis, On the holonomy group of linear connections, Indagationes Math. vol. 15 (1953) pp. 233-249; vol. 16 (1954) pp. 17-25.

9. K. Nomizu, Invariant affine connections on homogeneous spaces, Amer. J. Math. vol. 76 (1954) pp. 33-65.

10. - Sur les transformations affine d'une variêté riemanniénné, C. R. Acad. Sci. Paris vol. 237 (1953) pp. 1308-1310.

11. - Applications de l'etude de transformation affine aux espaces homogeneous reimanniens, C. R. Acad. Sci. Paris vol. 237 (1953) pp. 1386-1387.

12. A. Lichnerowicz, Espaces homogenes kahleriens, Colloque International de Geometrie Differentielle, Strasbourg, 1953, pp. 171-184.

13. K. Yano and S. Bochner, Curvature and Bette numbers, Annals of Mathematics Studies, no. 32, Princeton, 1953.

Institute for Advanced Study,

Princeton, N. J. 